\title{
CNO abundances in Early Type Be Stars
}

Y. Frémat ${ }^{1}$, J. Zorec ${ }^{2}$, R. Levenhagen ${ }^{3}$, N. Leister ${ }^{3}$, A.-M. Hubert ${ }^{1}$, M. Floquet $^{1}$, and C. Neiner ${ }^{1}$

1 Observatoire de Paris, Section d'Astrophysique de Meudon, GEPI, FRE $K$ 2459, 5 Place Jules Janssen, 92195 Meudon CEDEX, France

${ }^{2}$ Institut d'Astrophysique de Paris, CNRS, 98bis Boulevard Arago, 75014 Paris, France

${ }^{3}$ Instituto de Astronomia, Geofisica e ciencias Atmosféricas, Brazil

\begin{abstract}
High dispersion spectra in the visible range were obtained for field Be stars in both hemispheres. As Be stars form a class of Main Sequence fast rotators, we are intended to test how their chemical composition can be affected by rotation.
\end{abstract}

\section{Introduction}

The effect of rotation mixing on the helium and CNO abundances has already been observed in several fast rotating main sequence $\mathrm{O}$ type stars (eg: Howarth et al. 2001). At lower masses, studies (Gies \& Lambert 1992) made on the atmospheric composition of B type stars show either that there is no clear evidence of such an effect on the carbon and nitrogen abundances or if it existed, it would not be so effective than expected from theory (Herrero \& Lennon 2002). It is worth noting that the stellar samples used in most of these studies were exclusively formed with low $v \sin i$ objects showing no emission in the optical spectrum and most of them probably are slow rotators. In the present contribution, we show preliminary results of a study of the CNO chemical composition in early type Be stars of an ongoing observation program with the FEROS (ESO) and AURELIE (OHP) spectrographs.

\section{Model Atmospheres}

The effects of gravitational darkening due to fast rotation were taken into account in the same way as in Frémat et al. (2002) where the stellar surface is represented by a mesh of plane parallel atmosphere models. The local model atmospheres we used are those of Kurucz (1994) computed with ATLAS9 assuming LTE, but the level populations of the atoms and ions of interest were computed in NLTE using the TLUSTY computer code (Hubeny \& Lanz, 1995). 


\section{Discussion}

Fundamental parameters and abundances are determined fitting the optical spectra. Resulting carbon to nitrogen ratios are reported in Fig.1 against the oxygen abundance for each of the 4 studied stars (filled squares). In the same figure we represented the average $[\mathrm{C} / \mathrm{N}]$ level observed in the nitrogen normal stars analyzed by Gies \& Lambert (1992) and the one predicted by theory for a B type star of 9 to 12 solar masses with initial equatorial rotation speed of 300 $\mathrm{km} \mathrm{s}^{-1}$ (Meynet \& Maeder 2000).

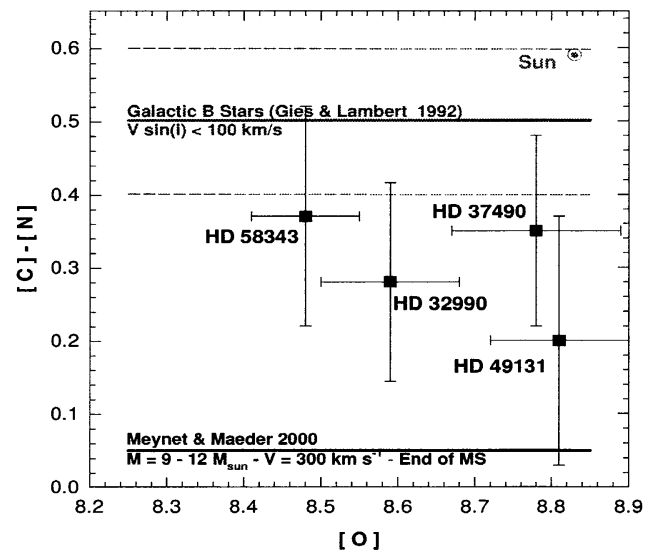

Figure 1. Abundance ratio of carbon to nitrogen $[\mathrm{C} / \mathrm{N}]$

It is expected that the location of Be stars in this figure depends on their age and on their initial angular speed. Our sample is quite small and the relative ages of the objects are not significatively different, however it is remarkable that each $\mathrm{Be}$ star we observed have $[\mathrm{C} / \mathrm{N}]$ ratios that are systematically lower than the average value that is observed in slow rotating $\mathrm{B}$ type stars. This implies a lower carbon to nitrogen abundance that could be attributed to rotation mixing.

Acknowledgments. This work has been supported by a Marie Curie Individual Fellowship under contract number HPMF-CT-2000-00497.

\section{References}

Gies, D. R. \& Lambert, D. L. 1992, ApJ 387, 673

Frémat, Y., Zorec, J., Hubert, A.-M., Floquet, M., Leister, N., Levenhagen, R., Chauville, J. \& Ballereau, D. 2003, in: A. Maeder \& P. Eenens (eds.), Stellar Rotation, Proc. IAU Symp. No. 215 (San Francisco: ASP)

Herrero, A. \& Lennon, D. 2003, in: A. Maeder \& P. Eenens (eds.), Stellar Rotation, Proc. IAU Symp. No. 215 (San Francisco: ASP)

Howarth, I.D. \& Smith, K.C. 2001, MNRAS 327, 353

Hubeny, I. \& Lanz, T. 1995, ApJ, 439, 875

Kurucz, R. 1994, CD-ROM No. 19.

Meynet, G. \& Maeder, A. 2000, A\&A 361, 101 